\title{
Missed Diagnosis of an Intraorbital Foreign Body of Homemade Fireworks Origin: A Case Report
}

\author{
Dan-dan Zhou ${ }^{a} \quad K_{a i} \mathrm{Niu}^{\mathrm{b}}$ Cheng-wei $\mathrm{Lu}^{\mathrm{c}}$ Ji-long Hao \\ Bing-jie Zhang ${ }^{c}$ Peng Hui ${ }^{c}$ \\ Departments of ${ }^{\mathrm{a}}$ Radiology, ${ }^{\mathrm{b}}$ Otorhinolaryngology and ${ }^{\mathrm{c} O p h t h a l m o l o g y}$, the First Hospital \\ of Jilin University, Changchun, China
}

\section{Key Words}

Homemade fireworks · Maxillofacial and orbital trauma · Orbital foreign body $\cdot$ Retinal detachment

\begin{abstract}
Purpose: We report a rare case of traumatic injury to the eye caused by homemade fireworks in a Chinese juvenile patient with a metal ring left in the orbit after having been sutured at the Emergency Department. Methods: An 11-year-old boy presented with a traumatic injury to the right eye from homemade fireworks. Following initial assessment involving maxillofacial computed tomography (CT) and suturing at the Emergency Department, he was transferred to our department for further evaluation because of his poor sight 1 day later. On examination, a skin laceration beneath the right eyebrow was noted, but the superior orbit was not fully visible on the maxillofacial CT performed 1 day previously. Therefore, an orbital CT scan was carried out on the second day, which showed a hyperdense ring embedded in the superior border of the orbital wall; the ring was surgically removed. On postoperative day 7, a fundus examination revealed resolving vitreous hemorrhage, blunt traumatic retinal detachment, and a large retinal tear superior to the macula. The patient refused to take surgery for retinal detachment into consideration. Therefore, we opted for oral steroids and careful observation. Results: After 2 months' observation, the large retinal tear had healed and white fibrous scar tissue had developed, and the retinal detachment superior to the macula had reattached itself spontaneously. The patient's vision had further improved to 20/200. During 1 year of follow-up, he remained clinically stable. Conclusion: To avoid missing the diagnosis, a complete history of the mechanism of injury and accurate imaging still prove most useful. Complete removal of the foreign body by the emergency physician is necessary because
\end{abstract}


Zhou et al.: Missed Diagnosis of an Intraorbital Foreign Body of Homemade Fireworks Origin: A Case Report

of the ocular toxicity of an iron-containing foreign body. As evidenced by the current case, oral steroids and observation for a period of several months is a management of choice for traumatic retinal detachment and retinal tear superior to the macula associated with homemade fireworks in children.

(C) 2015 The Author(s)

Published by S. Karger AG, Basel

\section{Background}

Although the use of homemade fireworks is banned by legislation in China, juvenile eye injuries from illegal homemade fireworks occur especially in the countryside. Few studies in the international literature have focused on homemade firework-related injuries. In the US, during June to July 2002, approximately $300(5.3 \%)$ of the patients with firework-related injuries were injured in incidents involving illegal and homemade fireworks in the 17 participating states in a study by the Centers for Disease Control and Prevention [1]. There are several reports on firework-related injuries [2-7]. Most of them happened in Iran during the annual Last Wednesday of the Year Festival (Charshanbeh Soori), and homemade grenades were the most frequent type of fireworks causing injury [2]. The severity of injuries varied greatly, and orbital foreign bodies may be difficult to diagnose clinically and radiologically due to the complicated composition of the homemade fireworks. Reliable imaging information is necessary to elucidate emergent injuries, to preoperatively plan the reconstruction of functional areas, and to guide the physical rehabilitation process. Computed tomography (CT) scan is considered the gold standard imaging technique for acute iron-containing orbital foreign bodies $[8,9]$. However, CT findings can be variable; to avoid missing the diagnosis, careful follow-up is needed. Herein, we report a rare case of traumatic injury to the eye from homemade fireworks in a Chinese juvenile patient with a metal ring left in the orbit after having been sutured at the Emergency Department.

\section{Case Presentation}

An 11-year-old boy presented with a traumatic injury to the right eye from homemade fireworks. Following initial assessment involving maxillofacial CT and suturing at the Emergency Department, he was transferred to our department for further evaluation because of his poor sight 1 day later. On examination, it was noted that several superficial wounds had been sutured before the child presented to us. His vision was limited to light perception, and the intraocular pressure was $21 \mathrm{~mm} \mathrm{Hg}$. A 60\% hyphema was present. A skin laceration beneath the right eyebrow was noted, but the superior orbit was not fully visible on the maxillofacial CT performed 1 day previously. Therefore, an orbital CT scan was carried out on the second day, which again showed a hyperdense ring (fig. 1a, arrow); this magnetic metal ring, measuring $18 \times 12 \mathrm{~mm}$ and embedded in the superior border of the orbital wall, was surgically removed (fig. 1 b, c). On postoperative day 7 , the blood clot in the anterior chamber had decreased in size, and a 1-mm-layered hyphema was present. The patient's visual acuity had improved to 20/400. A fundus examination revealed resolving vitreous hemorrhage, retinal hemorrhage (fig. 1d, short arrow), blunt traumatic retinal detachment superior to the macula (fig. 1d, long arrow), and a large retinal tear parallel to the corneoscleral limbus located in the nasal superior fundus quadrant. The patient and his parents refused to take surgery for retinal detachment into consideration. Therefore, we opted for starting on oral steroids for 1 month and careful observation. After 2 months' observation, discrete areas of 
Zhou et al.: Missed Diagnosis of an Intraorbital Foreign Body of Homemade Fireworks Origin: A Case Report

retinal pigment epithelial hypertrophy and atrophy, retinal hemorrhage, and epiretinal membrane formation (fig. 1e, short arrow) were seen in the right eye. The large retinal tear had healed and white fibrous scar tissue had developed (fig. 1e, long arrow), and the retinal detachment superior to the macula had reattached itself spontaneously. His vision had further improved to 20/200. During 1 year of follow-up, he remained clinically stable.

\section{Discussion}

Because of the complicated composition of homemade fireworks, the severity of injuries varies greatly. The first clinical and radiographic examination of the traumatized patient is crucial to determine the initial diagnosis, the severity of the injury, and a treatment plan as well as to create a baseline for follow-up. In the case of a patient who has undergone maxillofacial injury or orbital trauma associated with homemade fireworks, complete history taking and physical examinations must be performed. On suspicion of an iron-containing foreign body, an imaging study such as CT must be performed [8, 9]. However, because CT findings can be variable, exploratory surgery over the suspected area and careful follow-up is needed. A CT scan of the orbit helps to better delineate the location of the foreign body and its relation to the surrounding bony and parenchymal structures in an emergency situation. In this case, the initial maxillofacial CT did not show the foreign body in the orbit, because the scan region was not large enough. Thus, in the event of maxillofacial injury, especially with skin lacerations beneath the eyebrow, a CT scan of the orbit is useful to detect the foreign body in the orbit and to avoid misdiagnosis. Meanwhile, same-day ophthalmology referral is recommended.

Ocular toxicity of an iron-containing foreign body that is retained and even located in the orbit after an injury may produce ocular siderosis and severe progressive visual loss [10, 11]. Gerkowicz et al. [12] studied the penetration of iron and found that iron passes from the orbit into the eyeball and accumulates in considerable quantities in the sclera, choroid, retina, ciliary body, and even in the vitreous and corneal epithelium. Clinical manifestations include a rust-colored corneal stroma, heterochromia iridis, pupillary mydriasis, uveitis, cataract, lens subluxation, secondary glaucoma, and retinal degeneration. The electroretinogram may be normal or hypernormal early in the disease process, but it gradually decreases in amplitude as the disease progresses $[10,11]$. Therefore, an exploratory surgery over the suspected area and complete removal of the foreign body by the emergency physician is necessary before the wounds have been sutured.

The management of juvenile traumatic retinal detachment is still challenging for ophthalmic surgeons. The functional and anatomic outcomes of retinal detachment in children are less successful than in adults. Advanced proliferative vitreoretinopathy (PVR) as a surgical outcome in juvenile retinal detachment is more frequent than in adults. The frequency of advanced PVR in juvenile patients was $37.5 \%$ in a study by Akabane et al. [13] and 29.8\% in a study by Delage and Bonnet [14]. The reasons for the more frequent association of advanced PVR with juvenile retinal detachment may be not only a delayed diagnosis and prolonged retinal detachment but also a higher intraocular cellular activity. The frequency of a postoperative visual acuity worse than $20 / 200$ was 3 in the 10 patients younger than 12 years of age in the study by Akabane et al. [13]. As evidenced by the current case, nevertheless, spontaneous closure of a retinal tear and retinal reattachment can occur. It remains unclear whether surgical intervention could improve the visual outcomes. Oral steroids and observation for a period of several months is a management of choice for traumatic retinal detachment and tear superior to the macula associated with homemade fireworks in chil- 
Zhou et al.: Missed Diagnosis of an Intraorbital Foreign Body of Homemade Fireworks Origin: A Case Report

dren. Future studies with more cases might further improve our knowledge of this entity. Moreover, a strict legislation regarding the domestic use of homemade fireworks should be considered because of the increasing incidence of these injuries.

\section{Statement of Ethics}

Informed consent has been obtained from the subject, and the study protocol has been approved by the institute's committee on human research.

\section{Disclosure Statement}

None of the authors have any conflicts of interest regarding the material presented in this paper.

\section{References}

1 Centers for Disease Control and Prevention (CDC): Injuries associated with homemade fireworks - selected states, 1993-2004. MMWR Morb Mortal Wkly Rep 2004;53:562-563.

-2 Vaghardoost R, Ghavami Y, Sobouti B, Mobayen MR: Mortality and morbidity of fireworks-related burns on the annual Last Wednesday of the Year Festival (Charshanbeh Soori) in Iran: an 11-year study. Trauma Mon 2013;18:81-85.

-3 Pallantzas A, Kourakos P, Stampolidis N, Papagianni E, Balagoura A, Stathopoulos A, Polizoi A, Emvalomata A, Evaggelopoulou M, Castana 0: Burns during Easter festivities in Greece. Ann Burns Fire Disasters 2012;25:171-177.

4 Tavakoli H, Khashayar P, Amoli HA, Esfandiari K, Ashegh H, Rezaii J, Salimi J: Firework-related injuries in Tehran's Persian Wednesday Eve Festival (Chaharshanbe Soori). J Emerg Med 2011;40:340-345.

5 Saadat S, Naseripour M, Smith GA: The health and economic impact of fireworks-related injuries in Iran: a household survey following the New Year's Festival in Tehran. Injury 2010;41:e28-e33.

6 Mansouri MR, Mohammadi SF, Hatef E, Rahbari H, Khazanehdari MS, Zandi P, Moghimi S, Piri N, Fahim A: The Persian Wednesday Eve Festival 'Charshanbe-Soori' fireworks eye injuries: a case series. Ophthalmic Epidemiol 2007;14:17-24.

-7 Vassilia K, Eleni P, Dimitrios T: Firework-related childhood injuries in Greece: a national problem. Burns 2004;30:151-153.

-8 Lakits A, Prokesch R, Scholda C, Bankier A: Orbital helical computed tomography in the diagnosis and management of eye trauma. Ophthalmology 1999;106:2330-2335.

-9 Chen CS, Davis GJ, Selva D: Orbital foreign body misdiagnosed as superior orbital rim fracture. Clin Exp Ophthalmol 2002;30:295-296.

-10 Fineman MS, Sharma S, Shah GK, Brown GC, Eagle RC Jr: Ultrasound biomicroscopic diagnosis of an occult intrascleral foreign body: an unusual case of ocular siderosis. Retina 2001;21:265-267.

11 Weiss MJ, Hofeldt AJ, Behrens M, Fisher K: Ocular siderosis. Diagnosis and management. Retina 1997;17:105-108.

12 Gerkowicz K, Prost M, Wawrzyniak M: Experimental ocular siderosis after extrabulbar administration of iron. Br J Ophthalmol 1985;69:149-153.

13 Akabane N, Yamamoto S, Tsukahara I, Ishida M, Mitamura Y, Yamamoto T, Takeuchi S: Surgical outcomes in juvenile retinal detachment. Jpn J Ophthalmol 2001;45:409-411.

14 Delage S, Bonnet M: Microchirurgie des décollements de la rétine de l'enfant. J Fr Ophtalmol 1993;16:291296. 


\section{Case Reports in \\ Ophthalmology}

\begin{tabular}{l|l}
\hline Case Rep Ophthalmol 2015;6:448-452 \\
\hline DOI: 10.1159/000442584 & $\begin{array}{l}\text { c } 2015 \text { The Author(s). Published by S. Karger AG, Basel } \\
\text { www.karger.com/cop }\end{array}$
\end{tabular}

Zhou et al.: Missed Diagnosis of an Intraorbital Foreign Body of Homemade Fireworks Origin: A Case Report

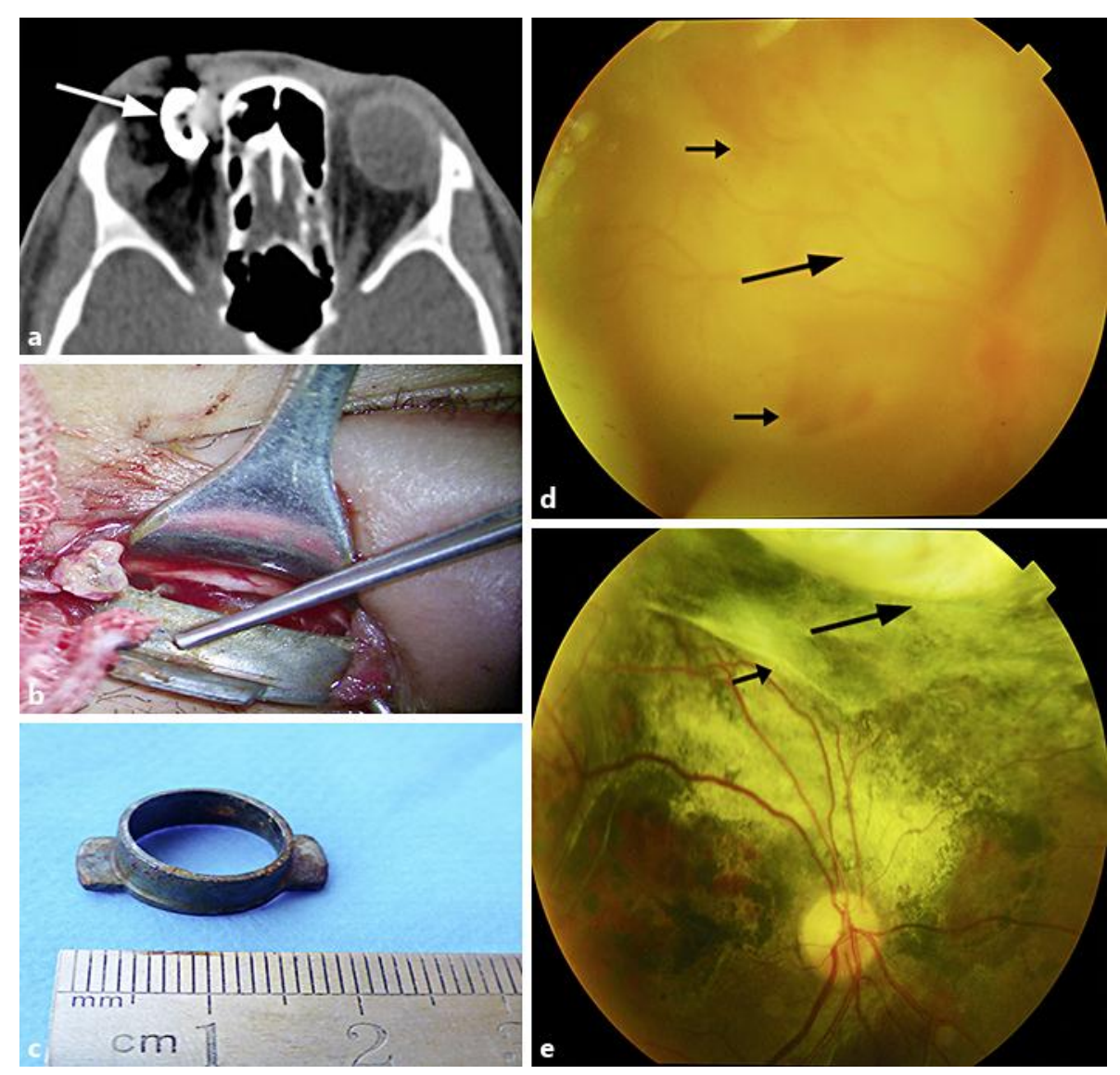

Fig. 1. An 11-year-old boy presented with a traumatic injury to the right eye for 1 day. a A CT scan of the orbit demonstrates the hyperdense ring. $\mathbf{b}$ The clinical photograph demonstrates the removal of a large intraorbital foreign body. c An $18 \times 12-\mathrm{mm}$ iron foreign body is extracted. $\mathbf{d}$ A funduscopic photograph on initial presentation reveals vitreous hemorrhage, retinal hemorrhage (short arrows), and blunt traumatic retinal detachment (long arrow) superior to the macula. e After 2 months' observation, a funduscopic photography shows retinal damage in a stable condition and the development of white fibrous scar tissue (short arrows), and the large retinal tear has healed (long arrow). 